\title{
Eye spy with my little eye: motivational relevance of visual stimuli guide eye- movements at different processing stages
}

Article

Accepted Version

Creative Commons: Attribution-Noncommercial-No Derivative Works 4.0

McSorley, E., Morriss, J. and Van Reekum, C. (2017) Eye spy with my little eye: motivational relevance of visual stimuli guide eye-movements at different processing stages. Biological Psychology, 123. pp. 8-14. ISSN 0301-0511 doi:

https://doi.org/10.1016/j.biopsycho.2016.11.008 Available at https://centaur.reading.ac.uk/68214/

It is advisable to refer to the publisher's version if you intend to cite from the work. See Guidance on citing.

Published version at: http://www.sciencedirect.com/science/article/pii/S030105111630357X

To link to this article DOI: http://dx.doi.org/10.1016/j.biopsycho.2016.11.008

Publisher: Elsevier

All outputs in CentAUR are protected by Intellectual Property Rights law, including copyright law. Copyright and IPR is retained by the creators or other copyright holders. Terms and conditions for use of this material are defined in the End User Agreement.

www.reading.ac.uk/centaur 
Central Archive at the University of Reading

Reading's research outputs online 
RUNNING HEAD: Motivation and Resource Allocation

Eye spy with my little eye: motivational relevance of visual stimuli guide eyemovements at different processing stages

\author{
Eugene McSorley ${ }^{1}$ \\ Jayne Morriss ${ }^{2}$
}

Carien M. van Reekum ${ }^{3}$

${ }^{1}$ Eugene McSorley (Corresponding Author), School of Psychology and Clinical Language Sciences, University of Reading, Reading, RG6 6AL, United

Kingdom. Email: e.mcsorley@reading.ac.uk.

2Jayne Morriss, Centre for Integrative Neuroscience and Neurodynamics, School of Psychology and Clinical Language Sciences, University of Reading, Reading, RG6 6AL, United Kingdom.

${ }^{3}$ Carien M. van Reekum, Centre for Integrative Neuroscience and Neurodynamics, School of Psychology and Clinical Language Sciences, University of Reading, Reading, RG6 6AL, United Kingdom. 
RUNNING HEAD: Motivation and Resource Allocation

\begin{abstract}
Visual stimuli may be selected for priority at different stages within the processing stream, depending on how motivationally relevant they are to the perceiver. Here we examine the extent to which individual differences in motivational relevance of task-irrelevant images (spider, crash, baby, food and neutral) guide eye-movements to a simple "follow the cross" task in 96 participants. We found affective images vs. neutral images to be generally more distracting, as shown by faster first saccade latencies and greater deviation in the final landing position from the target cross. The most arousing images (spider and food), compared to neutral images, showed the largest trajectory deviations of the first saccade. Fear of spiders specifically predicted greater deviation in the final landing position on spider images. These results suggest that attentional biases towards arousing and motivationally relevant stimuli may occur at different processing stages.
\end{abstract}

Keywords: attention, eye movements, threat, arousal, motivational relevance, fear of spiders 
RUNNING HEAD: Motivation and Resource Allocation

\section{Introduction}

From moment to moment, humans are confronted with a multitude of dynamic visual stimuli. However, because humans have limited selective attention, only a subset of stimuli can be focused on at any given time (Driver, 2001). Visual stimuli that capture attention more readily than others likely contain significant information for survival (LeDoux, 2000; Öhman \& Mineka, 2001; Brosch, Sander, Pourtois \& Scherer, 2008).

Initially, it was postulated that threat-related stimuli such as aggressive conspecifics and predatory animals were prioritized in attention over all other types of stimuli, as part of a fear system that has evolved to enable preconscious processing and immediate response (LeDoux, 2000; Öhman \& Mineka, 2001). For example, a wealth of data have shown that threat-related stimuli presented in a scene or among distractors are often found very quickly (Pflugshaupt et al., 2007; Rinck \& Becker, 2006; Soares, Esteves, \& Flykt, 2009; but see Lipp, Derakshan, Waters, \& Logies, 2004), they distract during search for a neutral target (Miltner, Krieschel, Hecht, Tripp, \& Weiss, 2004; Rinck, Reinecke, Ellwart, Heuer, \& Becker, 2005) and are generally more discriminable from a background than neutral targets (Öhman et al., 2001; Rinck et al., 2005; Larson et al., 2007). Furthermore, Lipp and Derakshan (2005) reported that even in participants low on spider fear, an attentional bias towards spider pictures was still present, suggesting a general mechanism of preferential processing of fear-relevant information.

More recently, the threat prioritization account has been countered by a number of studies suggesting attention to be captured by motivationally 
RUNNING HEAD: Motivation and Resource Allocation

relevant stimuli more generally (Brosch et al., 2008; Schupp, Junghöfer, Weike, \& Hamm, 2003). For example, studies in which both pleasant and unpleasant visual stimuli are presented demonstrate attentional modulation for both types of information. This effect is particularly strong when the information is highly arousing, such as images of mutilation, erotica, babies and food (e.g. Brosch, Sander, Pourtois \& Scherer, 2008; Schupp, Junghöfer, Weike, \& Hamm, 2003; Schupp et al., 2007), of particular interest to the participant e.g., Doctor Who fans (Purkis, Lester, \& Field, 2011) or relevant to the perceiver's current goals (e.g. Vogt et al., 2010). In short, it may not be the threat-relevant information per se that drives attentional capture, but the extent to which information is appraised as relevant to the perceiver.

Recently, a study by McSorley and Morriss (2015) pitted the threatprioritization and motivationally relevant accounts against each other by examining visual attention at different processing stages. Visual attention was assessed using a simple "follow the cross" task with flanking distractor images that varied in valence and arousal (e.g. babies, food, spiders and neutral). Individual differences in self-reported spider fear served as a grouping factor to assess the role of motivational relevance. Based upon previous studies of saccadic eye movements with non-emotional stimuli (McSorley, Cruickshank, \& Inman, 2009) and threat-related stimuli (Miltner et al., 2004; Pflugshaupt et al., 2007; Rinck \& Becker, 2006), eye movement metrics and dynamics were taken to reflect the real time, overt manifestation of a covert attentional system at different processing stages. For instance, first saccade latency is a temporal measure of initial attentional deployment. First saccade trajectory 
RUNNING HEAD: Motivation and Resource Allocation

and its subsequent landing position are spatial measures of initial attentional deployment. Second saccade latency is a temporal measure of attentional disengagement from the initial attentional location. McSorley \& Morriss (2015) found the spider-fearful and non-fearful group to show no difference in first saccade latency. Landing position deviations were greater for spiders and pleasant images for the spider-fearful group but only pleasant for the nonfearful group. Second saccade latencies were longer for the fearful than nonfearful group for spider images only. This pattern of results suggests that the impact of arousing images on saccade eye movements supports a general motivational relevance account rather than a specific threat-related priority.

Most of the findings supporting either a threat prioritization account or a motivational relevance account are based on behavioral measures of attentional bias, such as the dot-probe task or the visual search task. The measurement of eye movements supplements this work in important ways: 1) the real-time capture of overt attentional processes, and 2) the high resolution quantification of the time course of such processes. In the current study, we intended to replicate and extend McSorley and Morriss (2015) by examining people's eye movements while they completed a simple "follow the cross" task while distracting, task-irrelevant, arousing images were shown flanking the target cross. As before, we included arousing images depicting spiders and arousing but pleasant scenes (babies and food), but we also included threatrelevant but non-spider related scenes (e.g. a crash). Neutral images depicting common household objects served as control. A large crosssectional sample that varied in their fear of spiders took part and the impact of 
RUNNING HEAD: Motivation and Resource Allocation

task-irrelevant images on successful task completion was assessed.

Furthermore, addressing shortcomings in the McSorley and Morriss (2015) study, we collected ratings of valence and arousal for the images, and selfreported fear of spiders, state and trait anxiety, to assess coherence between ratings, questionnaires and eye movement metrics. Lastly, we directly compared the specificity of motivational relevance of fear of spiders against broader measures of anxious disposition upon ratings and eye movement metrics.

If motivationally relevant images are generally given priority of processing then their presence in the display environment should elicit quicker saccadic responses when compared with neutral images. Furthermore, if this priority for motivationally relevant images continues in the processing stream then they should be difficult to inhibit and deviations in saccade trajectory and landing position should be towards the distractor. This may extend to heightened engagement for motivationally relevant images with second saccade latencies being lengthened as disengagement from images with motivationally relevant content proves more difficult. Beyond this, if there is a specific processing priority we would expect this pattern to be related to individual differences in the extent of motivational relevance of the stimulus (e.g. fear of spiders). We expected spider images to elicit quicker responses and greater impact on saccade deviation as fear of spiders increases, i.e., saccades will be pulled towards spider images for more spider fearful people as they find these images more difficult to inhibit. Furthermore, we might expect the response time for the second saccade onset (the time difference 
RUNNING HEAD: Motivation and Resource Allocation

from the end of the first saccade to the onset of the second saccade) to be longer as the dispositional fear of spiders increases, as those who find spiders more fearful should find it more difficult to disengage from the spider stimuli, i.e., they fixate on spider fearful stimuli for longer. We further tested the specificity of self-reported fear of spiders, by comparing it with broader measures of anxiety, such as state and trait anxiety.

\section{Method}

\section{Participants}

96 people (86 females) with an age range of 18 to 41 took part. All observers had normal, or corrected to normal, vision and were recruited through the University or Reading's Psychology Department Research Panel for course credit, adverts placed around the campus area, and word of mouth. Local ethical approval was obtained and all participants gave their informed consent prior to inclusion.

\section{Materials}

\section{Questionnaires}

Self-reported spider fear was assessed on the Fear of Spiders Questionnaire (FSQ; Szymanski \& O'Donoghue, 1995) while trait and state anxiety were assessed using the State-Trait Anxiety inventory (STAI-X1 and STAI-X2; Spielberger, Gorsuch, Lushene Vagg \& Jacobs, 1983).

\section{Images}


RUNNING HEAD: Motivation and Resource Allocation

The 40 images used were largely taken from the International Affective Picture System (IAPS; Lang, Bradley \& Cuthbert, 2005; see appendix for IAPS numbers) with additional spider and food images found from a variety of sources, resulting in total in 10 threat-related pictures of various spiders (Spiders), 10 negative images of accidents (Crash), 10 positive images of food and babies (Food, Baby) and 10 neutral images of everyday objects such as chairs, tables (Neutral).

\section{Image Valence and Arousal Ratings}

To determine the affective value of the stimuli within this sample, subjective ratings of the stimuli were collected and, in line with the International Affective Picture System (IAPS), were rated on a 9-point Likert scale. Arousal ratings ranged from very calm (1) to very excited (9) and valence ratings ranged from very negative (1) to very positive (9). The images were presented in random order, in color using E-Prime software.

\section{"Follow the Cross" task}

Fixation and saccade targets were a cross (“+”), each line was $1^{\circ}$ in length. Targets were shown $8^{\circ}$ to the left or right of fixation on the horizontal meridian. A single image appeared either above or below the saccade target, the center of which was $2.1^{\circ}$ from the center of the target cross, with the nearest edge being $1^{\circ}$ away (See Figure 1 ). The centers of these images were at an angle of $27.5^{\circ}$ from the initial fixation point i.e., relatively "near" the target in order to 
RUNNING HEAD: Motivation and Resource Allocation

allow the distractor image to influence the saccade and for the extent of this to be modulated by its content. All images were $2.2^{\circ}$ by $2.93^{\circ}$ in size.

\section{Apparatus}

Eye movements were recorded using an Eyelink II eye-tracker with a sampling rate of $500 \mathrm{~Hz}$ (SR Research). Stimuli were presented on a 21" colour monitor with a refresh rate of $75 \mathrm{~Hz}$ (DiamondPro, Sony). Head movements were constrained with a chin-rest at a viewing distance of $1 \mathrm{~m}$. The eye-tracker was calibrated using a standard 9 point grid at the start of the experiment.
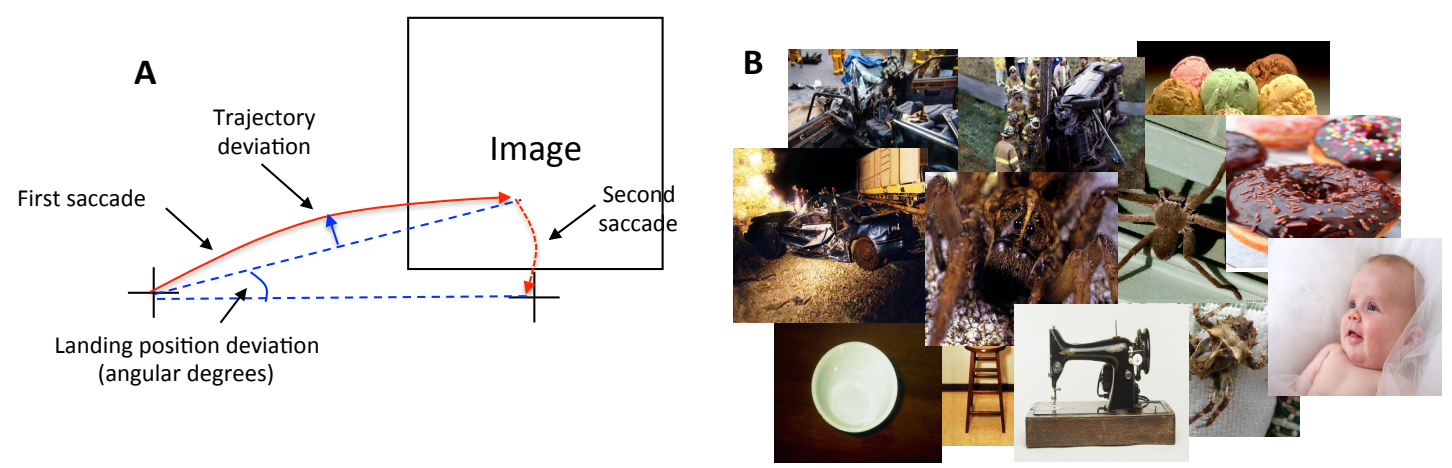

Figure 1: The layout for the experiment is shown in $(A)$. The target cross may appear on the left or the right of fixation on the horizontal meridian (here shown on the right). This was always accompanied by a flanking image which may be a Spider, Crash, Food, Baby or a Neutral image, examples of which are shown in (B). This image was shown above or below the target.

\section{Design and Procedure}

The experimental display consisted of a target cross to the left or right of fixation accompanied by a single image presented above or below its position. The onset of the experimental display was simultaneous to the offset 
RUNNING HEAD: Motivation and Resource Allocation

of the fixation screen. Each image was shown four times, giving a total of 180 trials. Trials began with a central fixation cross presented for a random duration between 800 and $1300 \mathrm{~ms}$. This was replaced by the onset of the experimental display, which stayed on for $1000 \mathrm{~ms}$. The experimental display was followed by a blank screen $(500 \mathrm{~ms})$ before the next trial. The task instruction to the participant was to move their eyes from the fixation cross to the target cross and to ignore the distracting images as best as they could.

\section{Eye Tracking Measures}

Saccade start- and endpoints were identified using a $22 \%$ selocity and $8000^{\circ} / \mathrm{s}^{2}$ acceleration criteria. The trajectory (maximum inflection point of the path) and landing position deviation (angular deviation) and the latency of the saccade were extracted for the first saccade response. Furthermore, in trials where a second corrective movement existed its latency was also extracted. The maximum trajectory deviation of each saccade relative to the direct path between fixation and landing position was determined (see Ludwig and Gilchrist, 2002) by fitting a second-order polynomial to the saccade path and finding the maximum point of angular deviation from the straight line that joined the saccade start position to its end position. Direction was defined as the angular deviation of saccade direction $\left(^{\circ}\right)$ taken from the initial fixation location to final endpoint in polar co-ordinates, with $0^{\circ}$ being a horizontal saccade. Positive values were assigned to deviations towards the critical images and negative values assigned when away. Averages were then 
RUNNING HEAD: Motivation and Resource Allocation

derived from this calculation. Latency was defined as the start of the saccadic movement relative to the onset of the experimental display.

Frist saccades were excluded from further analysis if saccade amplitude was more than $2^{\circ}$ from the target $(1.5 \%)$, response latency was quicker than 70 ms (classified as an anticipatory saccade) or slower than 500 ms (in these cases the saccade is taken as having not been driven by the experimental display) (0.57\%). Second saccades were not subject to this exclusion criteria short latency saccades could not be termed anticipatory but rather be evidence of parallel programming (Walker \& McSorley, 2006) and long latency saccades would be evidence of difficulties in disengaging. Data collected from each target position (left and right) and from each of the four possible image locations was collapsed. Therefore, each overall average represents data from a possible 40 trials.

\section{Results}

\section{Questionnaires}

The outcome of the scales show a wide range of ratings: FSQ: $M=44.71$, $S D=37.36$, range=0-119; STAI Trait: $M=37.84, S D=10.99$, range=14-70; STAI State: $M=40.69, S D=9.59$, range $=25-68$.

\section{Ratings}

Valence and arousal rating descriptive statistics are shown in Figure 2 and Table 1. Separate one-way ANOVA's were conducted on valence and arousal ratings with image type (Spider, Crash, Baby, Food and Neutral 
RUNNING HEAD: Motivation and Resource Allocation

images) as the factor. As expected, Spider and Crash images were rated as the most negative, compared to Baby, Food and Neutral images. Baby and Food images were rated as the most positive images, compared to Spider, Crash and Neutral images (overall ANOVA: $F(4,380)=333.293, \mathrm{MSE}=1.319$, $p<.0001, \eta^{2}=.778 ;$ All images vs. Neutral, $p$ 's < .05; All images vs. Spider, $p$ 's $<.05$; All images vs. Crash, $p$ 's $<.05$; All images vs. Baby, $p<.05$, except Baby vs. Food, $p=.065$; All images vs. Food, $p<.05$, except Food vs. Baby, $p=.065)$. Furthermore, Spider and Food images were rated as the most arousing, followed by Crash and Baby images, and lastly Neutral images (overall ANOVA: $F(4,380)=27.797, M S E=2.083, p<.0001, \eta^{2}=.226 ;$ All images, vs. Neutral, p's <.05; All images vs. Spider, p's <.05, except for Food vs. Spider, $p=.988$; All images vs Crash, $p$ 's $<.05$, except, Baby vs. Crash, $p=$ .168; All images vs Food, $p$ 's $<.05$, except Spider vs. Food, $p=.988$; All images vs Baby, $p$ 's $<.05$, except Crash vs. Baby $=.168)$.

To check that fear of spiders predicted valence and arousal ratings to Spider images, we carried out a hierarchical regression analysis. We created differences scores for both valence and arousal ratings of Spider, Crash, Food, Baby, compared to Neutral images (ratings from arousing images were subtracted from ratings to Neutral images, a positive difference score denotes higher relative arousal and higher relative pleasantness). Difference scores were entered as the dependent variable while general anxiety data was first entered into the model followed by FSQ. Trait and state anxiety at the first step did not predict valence ratings of Spider images $\left(R^{2}=.010, F<1\right)$, but as expected, including FSQ at the second step significantly improved the model 
RUNNING HEAD: Motivation and Resource Allocation

$\left(\Delta R^{2}=.079, F(1,89)=7.759, p=.007\right)$, such that higher $\mathrm{FSQ}$ scores predicted more negative ratings of Spider images. Furthermore neither trait and state anxiety nor FSQ significantly accounted for valence ratings of Crash, Food and Baby image types $\left(\Delta R^{2} s<.041, F^{\prime} s<2\right)$. Trait and state anxiety did predict arousal ratings of Spider images at the first step $\left(R^{2}=.311\right.$, $F(2,90)=4.889, p=.01)$, however, as predicted, the model was significantly improved by adding $\mathrm{FSQ}$ at the second step $\left(\Delta R^{2}=.072, F(1,89)=7.720\right.$, $p=.007$ ), such that higher FSQ scores predicted higher arousal ratings of Spider images. Neither trait and state anxiety, nor FSQ significantly accounted for arousal ratings of Crash, Food and Baby image types $\left(\Delta R^{2 ،} s<.009\right.$ F's<2.5).

A

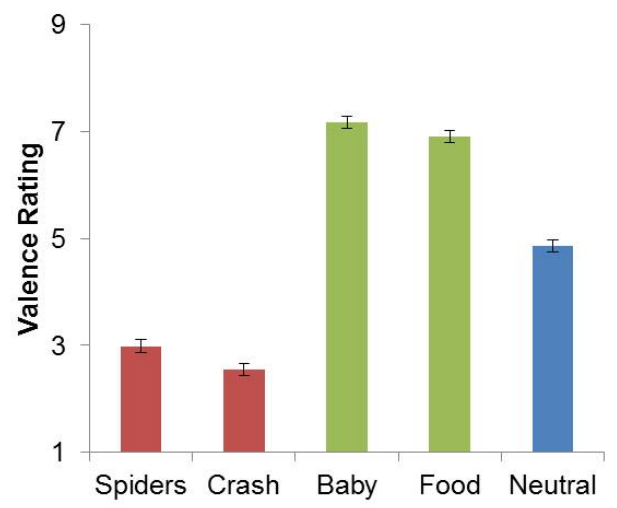

B 9

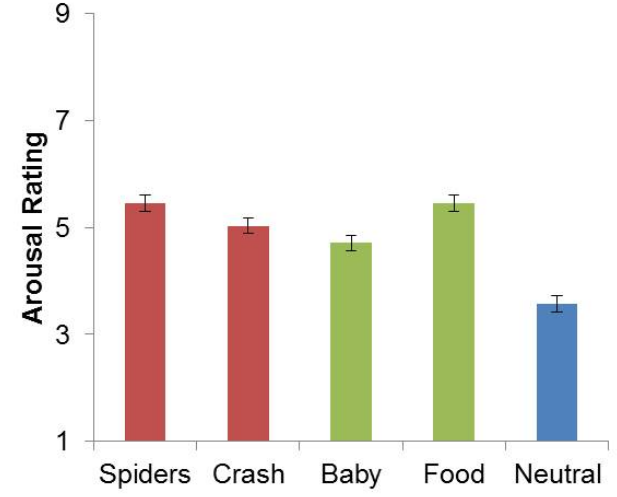

Figure 2: Bar graphs of Valence (A), and Arousal (B) ratings of the images used in the task.

For valence, $1=$ very negative, $9=$ very positive. For arousal, $1=$ calm, and $9=$ excited. Error bars are within subject 95\% confidence intervals (Loftus \& Masson 1994). 
A

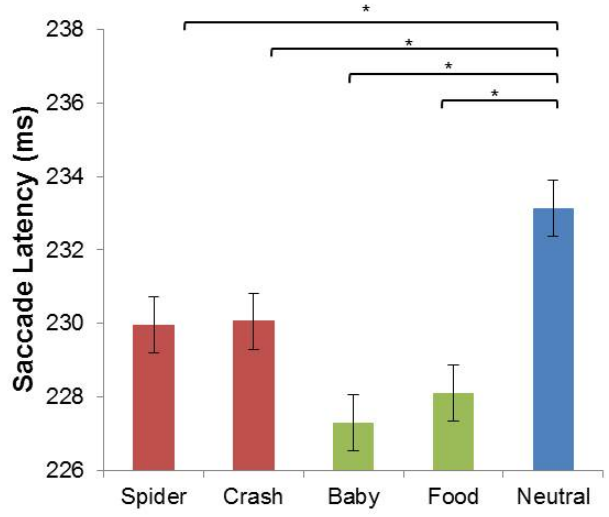

C

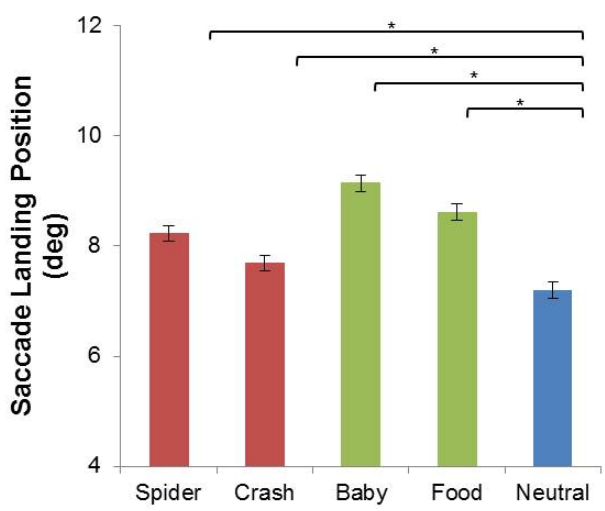

B

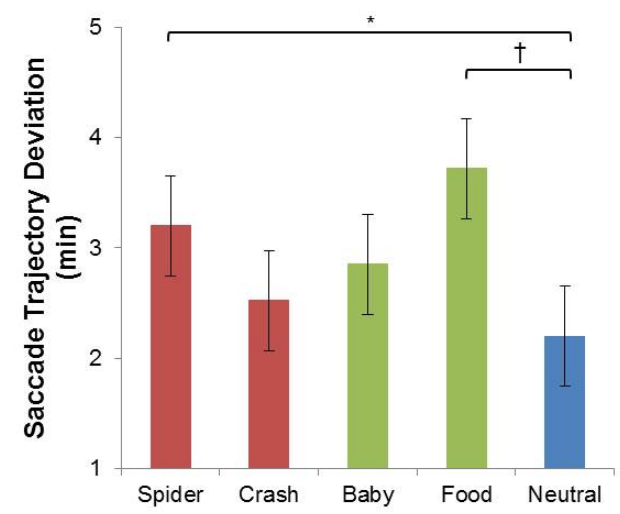

D

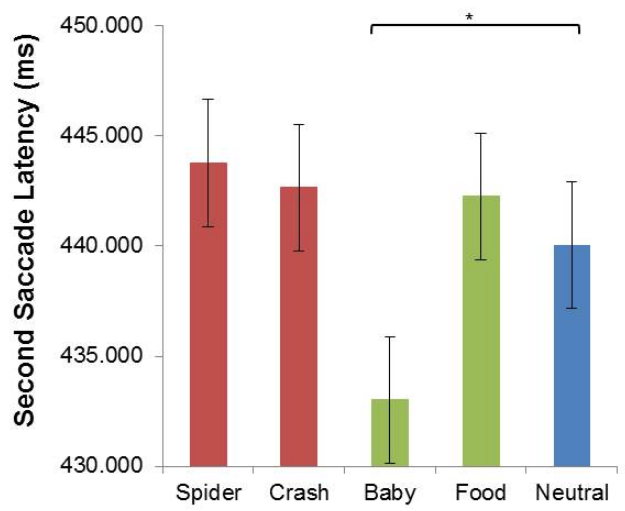

Figure 3: Shows the median saccade latency $(\mathrm{ms})(\mathrm{A})$, mean saccade trajectory $(B)$, mean landing position deviation of first saccades $(C)$ and the median latencies of the second saccades (D). Error bars are within subject 95\% confidence intervals (Loftus \& Masson 1994). ${ }^{*}=p<.05, \dagger=p<.06$.

\section{Eye-tracking Outcomes}

Descriptive statistics of the latency of the first saccades $(A)$, their average trajectory (B) and landing position (C) deviations and the second saccade latencies (D) are displayed in Figure 3 and Table 1. This pattern was examined with separate one-way ANOVA's for each measure with image type (Spider, Crash, Baby, Food and Neutral images) as the factor. These showed 
RUNNING HEAD: Motivation and Resource Allocation

that saccades were elicited more quickly towards all image types relative to Neutral (overall ANOVA: $F(4,380)=8.426$, MSE $=57.737, p<.0001, \eta^{2}=.081$; All images vs. Neutral, p's <.05). No overall significant effect was found for saccade trajectory deviations but planned contrasts showed that they deviated more greatly to Spider relative to Neutral images, with a strong trend towards significance also shown for the saccades elicited towards Food images deviating more strongly in their path relative to Neutral images (overall ANOVA: $F(4,380)=1.822, M S E=18.259, p=.124, \eta^{2}=.019$; Spider vs Neutral: $p=.031$; Food vs Neutral: $p=.057$; Baby or Crash vs Neutral: $p$ 's $>.161$ ) Landing position was found to deviate more greatly towards all images relative to Neutral images (overall ANOVA: $F(4,380)=26.962$, MSE $=2.075, p<.0001$, $\eta^{2}=.221 ;$ All images vs. Neutral, $p$ 's <.05). In contrast to this, second saccade latencies show an overall effect but this is driven by the disengagement from baby images being quicker than all other image types (overall ANOVA: $F(4,380)=2.970, \mathrm{MSE}=601.185, p=.019, \eta^{2}=.030$; Baby vs Neutral: $p<.05$; all other p's>.208).

In order to further assess the impact of the presence of Spider images as a function of general anxiety (both trait and state) and Fear of Spiders, we carried out a hierarchal regression analysis. First saccade latency, trajectory and landing position deviations and second saccade latencies were determined relative to Neutral images as a difference score (latencies and deviations elicited in presence of Spider images were subtracted from those elicited to Neutral images). These were entered as the dependent variable while trait and state anxiety data were first entered into the model followed by 
RUNNING HEAD: Motivation and Resource Allocation

FSQ. Neither trait and state anxiety nor fear of spiders were found to significantly account for the extent of saccade latency (STAI $R^{2}=.015, F<1$; FSQ $\Delta R^{2}=.000, F<1$ ), trajectory deviation (STAl $R^{2}=.004, F<1 ; \mathrm{FSQ} \Delta R^{2}$ $=.001, F<1$ ) or second saccade latency (STAI $R^{2}=.03, F<1 ; \mathrm{FSQ} \Delta R^{2}=.002$, $F<1)$. In contrast to this, while saccade landing position was not predicted by general anxiety in the first step of the model $\left(R^{2}=.005, F<1\right)$ it was significantly predicted by the addition of $\mathrm{FSQ}$ at the second step $\left(\Delta R^{2}=.072\right.$, $F(1,92)=7.184, p=.009)^{1}$.

\section{Concordance between ratings and eye-tracking outcomes}

We did not find image ratings and eye-tracking measure difference scores (e.g. spider - neutral) to significantly correlate, $p$ 's $>.05$.

${ }^{1}$ The same regression procedure was carried out separately for both saccade trajectory and landing position deviations made when images of Crashes, babies or Food were present. General anxiety or fear of spiders were found to not account for saccade trajectory deviations for any other image type (all p's>.372 except for FSQ and crash images which show a marginal trend: $\Delta r 2=.03, F(1,92)=2.828, p=.096)$. In contrast to this, saccade landing position deviation for images of Food but not Crashes or Babies did show a significant relationship with general anxiety $(r 2=.064, F(2,93)=3.158$, $p=.047$, other p's>.225) while FSQ showed no significant additional improvement to this $(\Delta \mathrm{r} 2=.026, \mathrm{~F}(1,92)=2.624, \mathrm{p}=.109$; all other $\mathrm{p}$ 's $>573)$. Both general anxiety and FSQ did not account for the first or second saccade latencies for all other image types. 
RUNNING HEAD: Motivation and Resource Allocation

\section{Discussion}

To examine whether generally motivationally relevant stimuli are given processing priority, or whether attentional capture is more specifically tied to arousing threat-related stimuli, participants were asked to saccade to a cross that could appear to the left or right of fixation while ignoring a task irrelevant distractor that could appear above or below the target. The distractor was an unpleasant threat-related stimulus (a Spider, more arousing and unpleasant to spider fearful participants), an unpleasant image or a pleasant image (e.g., Crash, Baby or Food, potentially arousing to all participants), or a nonarousing Neutral image.

The results show that images with emotional content, either pleasant or unpleasant, were judged to be more arousing than the neutral images, similar to the normative ratings provided as part of the IAPS (Lang, Bradley \& Cuthbert, 2005). Furthermore, in line with research supporting the motivational relevance account (Brosch, Sander, Pourtois \& Scherer, 2008; Schupp, Junghöfer, Weike, \& Hamm, 2003; Schupp et al., 2007), saccade responses on trials with distracting emotional content present were quicker than when a neutral image was present i.e., first saccade latencies were shorter. This suggests that the emotional content of an image is initially processed more rapidly and leads to stronger activation at the distractor image location than neutral images and this causes a shorter latency response in the eye movements. 
RUNNING HEAD: Motivation and Resource Allocation

Spider and Food images, not Crash and Baby images, show a deviation in the trajectory of the saccade towards them which was greater than that elicited by the presence of a Neutral image. This can be interpreted as being the result of activation at the distractor image location when Spider and Food images are present being higher than when the Neutral is present causing the saccade to be pulled towards their location. The extent of this deviation is not related to levels of anxiety or fear of spiders. Tentatively, we can suggest that this pattern of deviation in saccade trajectory was larger for Spider and Food images because they were the most arousing images to our participants, as shown in the ratings, which were similar to those shown in the original rating study (Lang, Bradley \& Cuthbert, 2005). However, the saccade trajectory findings are at trend and further work is needed to disentangle the effects of arousal on this measure.

Following this, all emotional image types show a greater magnitude in the deviation of the final landing position of the first saccade. This shows that the activation at the distractor image location was greater for all images with emotional content than the Neutral image at the end of the saccade. For Spider images, the magnitude of this deviation was found to be specifically related to fear of spiders, over and above trait and state anxiety: saccade landing position deviated toward Spider images more as fear of spiders was greater. Furthermore, we found fear of spiders to specifically predict valence and arousal ratings of Spider images over and above trait and state anxiety, in line with prior work finding fear of spiders to be part of an integrated set of animal fears, independently of trait anxiety (Davey, 1991). Overall, these 
RUNNING HEAD: Motivation and Resource Allocation

findings sit alongside previous work suggesting that stimuli which are more motivationally relevant to some individuals (e.g. more arousing and unpleasant for spider fearful individuals) can also capture attention (McSorley \& Morriss, 2015; Purkis, Lester, \& Field, 2011) and that particularly the landing position deviation may reflect this most directly of all eye movement variables.

Only Baby images show a shortening of second saccade latency. This suggests a quicker disengagement from that image type. We might have expected to find longer second saccade latencies reflecting a difficulty in disengagement from Baby images but also perhaps Crash and Food images and those that were motivationally relevant such as Spiders for those scoring higher on the FSQ. However, little impact of image type on second saccade latency was found. This suggests that, generally, attentional disengagement was not affected by the motivationally relevant image content in this case.

To summarize, the pattern of first saccade latencies and deviations both in saccade trajectory and landing position suggest that activation at the distractor location when emotional images are shown was initially more difficult to inhibit than Neutral images with rapid processing of arousing emotional content leading to shorter first saccade latencies. This difficulty continues for those images rated most arousing, Spider and Food images, as activity associated with those is higher than caused by the presence of the Neutral image, hence greater trajectory and landing position deviation towards those images. Crash and Baby images show no trajectory deviation differences with Neutral but do show a stronger deviation in saccade landing position, suggesting that after initial rapid processing, activation caused by the 
RUNNING HEAD: Motivation and Resource Allocation

emotional images is matched by that elicited by the Neutral image. However, for the emotional images activation continues to rise as shown by the deviation in the landing position of the first saccade toward those images relative to Neutral. The schematic in Figure 4 shows how this interpretation looks in terms of the saccadic movements recorded.

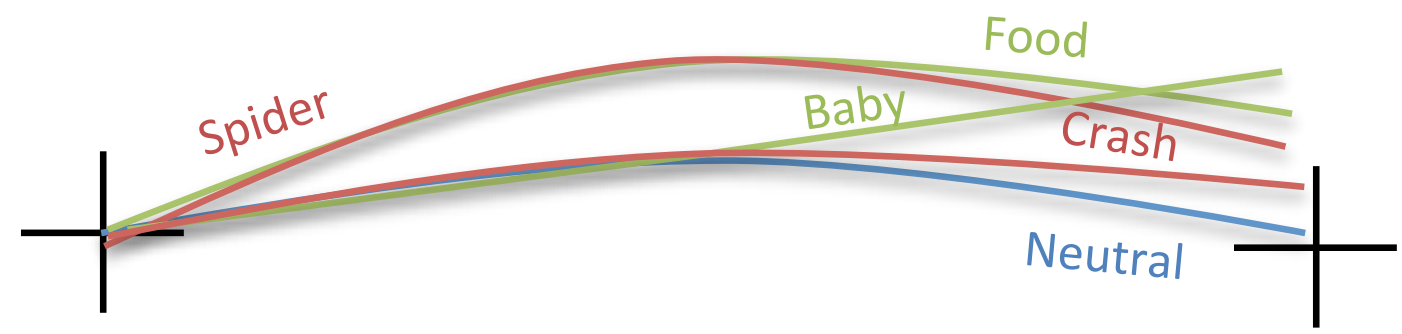

Figure 4: Shows a schematic of pattern of trajectory and landing positions for first saccades suggested from the analysis. Relative to the Neutral image both Spider and Food are initially difficult to inhibit and hence the saccade trajectory deviates towards their location. Inhibition of these images continues to be difficult, as does the inhibition of Crash and Food images and the deviation of the final saccade landing position is toward the images.

The pattern of results reported here is broadly similar to results presented by McSorley \& Morriss (2015) in which the same task was carried out but where a limited participant sample taken from the extremes of the reported FSQ scores, and a smaller set of image types (Threat-related Spiders, Pleasant and Neutral) were included. They also recorded less information about the sample and their responses to each image (e.g., arousal rates, anxiety questionnaires). Both this study and McSorley \& Morriss report greater effects of motivationally relevant (vs neutral) images on the saccade metrics of the trajectory and landing position deviations, supporting their initial interpretation of early attentional capture and resource allocation interruption 
RUNNING HEAD: Motivation and Resource Allocation

followed by sustained attention maintenance. In contrast, however, here we find evidence of quicker first saccade responses not seen by McSorley \& Morriss. This can be taken as further support for an initial capture of attention by emotionally relevant images (compared to neutral ones), but one that is not consistent across experiments. In contrast to earlier reports from our group (McSorley \& van Reekum, 2013) where saccade deviations towards the distractor were specific to unpleasant images, McSorley and Morriss (2015) report effects of motivational relevance on saccade control of trajectory and landing position. Our work presented here replicates these findings and suggest that the McSorley \& van Reekum (2013) findings may have been specific to the image set - pleasant images particularly - used in that study. Finally, in the experiment reported here we find limited effects on second saccade latencies whereas as McSorley \& Morriss found evidence that arousing images produced longer second saccade latencies. This suggests that difficulty with disengagement from motivationally relevant stimuli is not a consistent facet of this process, and may vary alongside the stimulus sets included.

Overall, this pattern of results support a wider interpretation of processing priority to one in which privileged access to processing resources are not just given the threat related stimuli (LeDoux, 2000; Öhman \& Mineka, 2001) but rather any motivationally relevant stimulus can be allocated immediate access to current resources (e.g. Brosch, et al., 2008). Our results sit well within the broad context of, and may be interpreted within, a "waves" of processing account (Pessoa \& Adolphs, 2010). Within this account we 
RUNNING HEAD: Motivation and Resource Allocation

suggest that an early and coarse processing of distractors takes place that is followed by a later, more detailed, processing. These impact differently on initial saccade response, its subsequent path and landing position deviation and disengagement depending on the priority of the motivationally relevant stimuli at different points in the processing stream. Our results show that all motivationally relevant stimuli may capture initial attention with faster first saccade response times and consistently show differences in the deviation towards the images in saccade trajectories are shown for the most arousing stimuli (Spider and Food). In contrast, capture of attention is shown via landing position deviations for all motivationally relevant stimuli. Furthermore, the extent of landing deviation to Spider images is determined by individual differences in the extent of motivational relevance e.g. those who find spiders more arousing and unpleasant have the largest saccade landing position deviation. We have previously found evidence for a difference in second saccade latencies across all those that are motivationally relevant or those which are more specifically threat-related but this is not supported by the results from the experiment reported here. Our wider suggestion is that the automatic allocation of attention takes place on the basis of motivational relevance rather than being specific to threat-relevant information.

These findings have important implications for when processes in the automatic allocation of attention go wrong. Processing biases that are maladaptive for threat have been suggested to have a causal role in the development of anxiety disorders and are implicated in the development and maintenance of various types of psychopathology (Cisler \& Koster, 2010; 
RUNNING HEAD: Motivation and Resource Allocation

Harvey, Watkins, Mansell, \& Shafran, 2004). The findings here suggest, in part, that such maladaptation of attention biases may not just be limited to the processing of threat-relevant information but also to other types of stimuli that likely elicit arousal, depending on the stage of attentional processing. We found that all emotion-relevant stimuli took precedence of earlier attentional processing, which is then driven by those that are rated as most arousing, whilst landing position, another metric of earlier attentional processing, was specifically affected by individual differences in the extent of motivational relevance. Later attentional biases (difficulty disengaging) were not observed in this study. Such findings may have implications for other types of motivationally relevant stimuli and samples, e.g., attentional biases for food in eating behavior and in extreme cases, eating disorders.

Acknowledgements: The authors wish to thank Victoria Cole, Charlotte Hopkins, Esther Hughes, Elizabeth Kidd and Cathryn Pitfield for their help in data collection. This research was supported by a Medical Research Council (MRC) doctoral studentship (MR/J003980/1) awarded to Jayne Morriss. For inquiries about access to the data, please contact Eugene McSorley.

\section{References}

Brosch, T., Sander, D., Pourtois, G., \& Scherer, K. R. (2008). Beyond fear. Psychological Science, 19(4), 362.

Cisler, J. M., \& Koster, E. H. W. (2010). Mechanisms of attentional biases 
RUNNING HEAD: Motivation and Resource Allocation

towards threat in anxiety disorders: An integrative review. Clinical Psychology Review, 30(2), 203-216. doi:10.1016/j.cpr.2009.11.003

Davey, G. (1991). Characteristics of individuals with fear of spiders. Anxiety Research, 4(4), 299-314.

Driver, J. (2001). A selective review of selective attention research from the past century. British Journal of Psychology, 92(1), 53-78.

Harvey, A., Watkins, E., Mansell, W., \& Shafran, R. (2004). Cognitive behavioural processes across psychological disorders: a transdiagnostic approach to research and treatment, Oxford University Press: Oxford, UK

Lang, P. J., Bradley, M. M., \& Cuthbert, B. N. (2005).International Affective Picture System (IAPS): Affective ratings of pictures and instruction manual (Tech. Report A-6). Gainesville: University of Florida, Center for Research in Psychophysiology.

Larson, C. L., Aronoff, J., \& Stearns, J. J. (2007). The shape of threat: simple geometric forms evoke rapid and sustained capture of attention. Emotion, 7(3), 526-534.

LeDoux, J. E. (2000). Emotion circuits in the brain. Annual Review of Neuroscience, 23, 155-184.doi:10.1146/annurev.neuro.23.1.155

Lipp, O. V., Derakshan, N., Waters, A. M., \& Logies, S. (2004). Snakes and Cats in the Flower Bed: Fast Detection Is Not Specific to Pictures of Fear-Relevant Animals. Emotion, 4(3), 233-250. doi:10.1037/15283542.4.3.233 
RUNNING HEAD: Motivation and Resource Allocation

Loftus, G. R., \& Masson, M. E. (1994). Using confidence intervals in withinsubject designs. Psychonomic Bulletin \& Review, 1(4), 476-490. http://doi.org/10.3758/BF03210951

McSorley, E., Cruickshank, A., \& Inman, L. (2009). The development of the spatial extent of oculomotor inhibition. Brain research, 1298, 92-98.

McSorley, E. \& Morriss, J. (2015). What you see is what you want to see: motivationally relevant stimuli can interrupt current resource allocation. Cognition \& Emotion. doi: 10.1080/02699931.2015.1081872

McSorley, E., \& van Reekum, C. (2013). The time course of implicit affective picture processing: an eye movement study. Emotion, 13, 769-773.doi: $10.1037 / \mathrm{a} 0032185$

Miltner, W. H. R., Krieschel, S., Hecht, H., Trippe, R., \& Weiss, T. (2004). Eye Movements and Behavioral Responses to Threatening and Nonthreatening Stimuli During Visual Search in Phobic and Nonphobic Subjects. Emotion, 4(4), 323-339. doi:10.1037/1528-3542.4.4.323

Öhman, A., \&Mineka, S. (2001). Fears, phobias, and preparedness: Toward an evolved module of fear and fear learning. Psychological Review, 108(3), 483-522. doi:10.1037//0033-295X.108.3.483

Öhman, A., Flykt, A., \&Esteves, F. (2001). Emotion drives attention: Detecting the snake in the grass. Journal of Experimental Psychology: General, 130(3), 466.

Pessoa, L., \&Adolphs, R. (2010). Emotion processing and the amygdala: from a 'low road" to "many roads" of evaluating biological significance, 1-10. doi:10.1038/nrn2920 
RUNNING HEAD: Motivation and Resource Allocation

Pflugshaupt, T., Mosimann, U., Schmitt, W., Wartburg, von, R., Wurtz, P., \&Luthi, M.. (2007). To look or not to look at threat? Scanpath differences within a group of spider phobics. Journal of anxiety disorders, 21(3), 353-366.

Purkis, H. M., Lester, K. J., \& Field, A. P. (2011). But what about the Empress of Racnoss? The allocation of attention to spiders and Doctor Who in a visual search task is predicted by fear and expertise. Emotion, 11(6), 1484-1488. doi:10.1037/a0024415

Rinck, M., \& Becker, E. S. (2006). Spider fearful individuals attend to threat, then quickly avoid it: Evidence from eye movements. Journal of abnormal psychology, 115(2), 231-238. doi:10.1037/0021$843 X .115 .2 .231$

Rinck, M., Reinecke, A., Ellwart, T., Heuer, K., \& Becker, E. S. (2005). Speeded Detection and Increased Distraction in Fear of Spiders: Evidence From Eye Movements. Journal of abnormal psychology, 114(2), 235-248. doi:10.1037/0021-843X.114.2.235

Schupp, H. T., Junghöfer, M., Weike, A. I., \& Hamm, A. O. (2003). Attention and emotion: an ERP analysis of facilitated emotional stimulus processing. Neuroreport, 14(8), 1107-1110. doi:10.1097/01.wnr.0000075416.59944.49

Schupp, H. T., Stockburger, J., Codispoti, M., Junghöfer, M., Weike, A. I., \& Hamm, A. O. (2007). Selective visual attention to emotion. The Journal of Neuroscience, 27(5), 1082-1089. 
RUNNING HEAD: Motivation and Resource Allocation

Soares, S. C., Esteves, F., \&Flykt, A. (2009). Fear, but not fear-relevance, modulates reaction times in visual search with animal distractors. Journal of anxiety disorders, 23(1), 136-144.

doi:10.1016/j.janxdis.2008.05.002

Szymanski, J., \&O'Donohue, W. (1995). Fear of Spiders

Questionnaire.Journal of behavior therapy and experimental psychiatry, 26(1), 31-34.

Vogt, J., De Houwer, J., Moors, A., Van Damme, S., \& Crombez, G. (2010).

The automatic orienting of attention to goal-relevant stimuli. Acta Psychologica, 134(1), 61-69.

Walker, R., \& McSorley, E. (2006). The parallel programming of voluntary and reflexive saccades. Vision Research, 46(13), 2082-2093. 
RUNNING HEAD: Motivation and Resource Allocation

Table 1.

Summary of means, medians (latency measures only) and standard deviations (SD) for each dependent measure as a function of image type

\begin{tabular}{lccccc}
\hline \hline & \multicolumn{5}{c}{ Image Type } \\
\cline { 2 - 5 } Measure & Spider & Crash & Baby & Chocolate & Neutral \\
\hline & & & & & \\
Eye-tracking & & & & & \\
Saccade Latency & 229.96 & 230.06 & 227.29 & 228.10 & 233.13 \\
(ms) & $(22.84)$ & $(24.96)$ & $(25.35)$ & $(25.34)$ & $(24.94)$ \\
Saccade Trajectory & 3.2 & 2.52 & 2.85 & 3.72 & 2.2 \\
Deviation (min) & $(6.32)$ & $(6.37)$ & $(6.64)$ & $(8.56)$ & $(6.06)$ \\
Saccade Landing & 8.23 & 7.69 & 9.15 & 8.62 & 7.20 \\
Position (deg) & $(4.41)$ & $(4.50)$ & $(4.68)$ & $(4.68)$ & $(4.36)$ \\
& & & & & \\
Second Saccade & 443.77 & 442.65 & 433.02 & 442.25 & 440.04 \\
Latency (ms) & $(60.14)$ & $(57.60)$ & $(57.02)$ & $(64.06)$ & $(52.64)$ \\
& & & & & \\
Ratings & & & & & \\
Valence & & & & & \\
& $(1.27)$ & $(1.07)$ & $(1.33)$ & $(1.10)$ & $(0.94)$ \\
Arousal & & & & \\
& 5.45 & 5.03 & 4.71 & 5.45 & 3.57 \\
& $(1.55)$ & $(1.37)$ & $(1.91)$ & $(1.73)$ & $(1.62)$ \\
\hline \hline
\end{tabular}

Note: Saccade latency measured in milliseconds; Saccade trajectory deviation measured in minute of arc; Saccade landing position measured in degrees; Second saccade latency measured in milliseconds. Ratings: For valence, $1=$ very negative, $9=$ very positive; For arousal, $1=$ calm, and $9=$ excited. 
RUNNING HEAD: Motivation and Resource Allocation

\section{Appendix}

Reference Numbers for Images Taken From the International (Lang, Bradley, \& Cuthbert, 2005)

$\begin{array}{lll}\text { Picture Type } & \text { Valence } & \text { IAPS Reference Number } \\ \text { Spider } & \text { Negative } & 1200 \\ \text { Spider } & \text { Negative } & 1201 \\ \text { Spider } & \text { Negative } & 1205 \\ \text { Spider } & \text { Negative } & 1220 \\ \text { Spider } & \text { Negative } & 1230 \\ \text { Spider } & \text { Negative } & 1240 \\ \text { Accident } & \text { Negative } & 9900 \\ \text { Accident } & \text { Negative } & 9901 \\ \text { Accident } & \text { Negative } & 9902 \\ \text { Accident } & \text { Negative } & 9903 \\ \text { Accident } & \text { Negative } & 9910 \\ \text { Accident } & \text { Negative } & 9911 \\ \text { Accident } & \text { Negative } & 9912 \\ \text { Accident } & \text { Negative } & 9920 \\ & & \\ \text { Accident } & \text { Negative } & 9921 \\ \text { Accident } & \text { Negative } & 9925 \\ \text { Baby } & \text { Positive } & 2070 \\ \text { Baby } & \text { Positive } & 2150 \\ \text { Baby } & \text { Positive } & 2057 \\ \text { Baby } & \text { Positive } & 2058 \\ \text { Food } & \text { Positive } & 7470 \\ \text { Food } & \text { Positive } & 7282 \\ \text { Food } & \text { Positive } & 7330\end{array}$

